\title{
Pyroelectric effect and polarization instability in self-assembled diphenylalanine
}

microtubes

A. Esin, I. Baturin, T. Nikitin, S. Vasilev, F. Salehli, V. Ya. Shur, and A. L. Kholkin’

Citation: Appl. Phys. Lett. 109, 142902 (2016); doi: 10.1063/1.4962652

View online: http://dx.doi.org/10.1063/1.4962652

View Table of Contents: http://aip.scitation.org/toc/apl/109/14

Published by the American Institute of Physics

\section{Articles you may be interested in}

Evidence of ferroelectricity and phase transition in pressed diphenylalanine peptide nanotubes Appl. Phys. Lett. 100, 043702043702 (2012); 10.1063/1.3676417

Ferroelectric, pyroelectric, and piezoelectric properties of a photovoltaic perovskite oxide Appl. Phys. Lett. 110, 063903063903 (2017); 10.1063/1.4974735

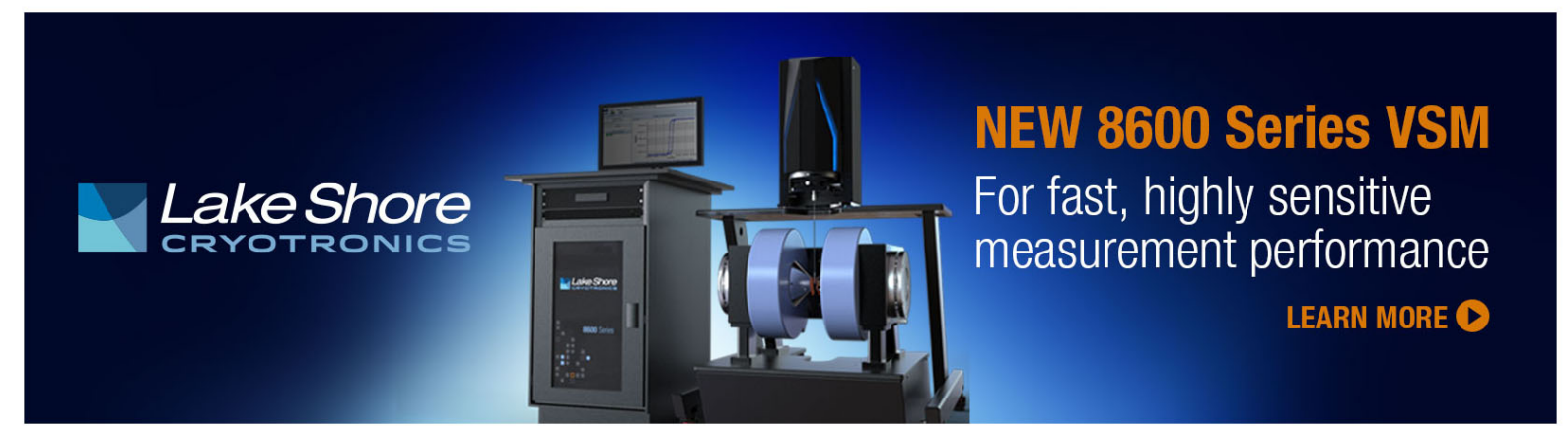




\title{
Pyroelectric effect and polarization instability in self-assembled diphenylalanine microtubes
}

\author{
A. Esin, ${ }^{1}$ I. Baturin, ${ }^{1}$ T. Nikitin, ${ }^{1}$ S. Vasilev, ${ }^{1}$ F. Salehli, ${ }^{2}$ V. Ya. Shur, ${ }^{1}$ and A. L. Kholkin ${ }^{1,3, a)}$ \\ ${ }^{1}$ Institute of Natural Sciences and Mathematics, Ural Federal University, 620000 Ekaterinburg, Russia \\ ${ }^{2}$ Department of Physics Engineering, Istanbul Technical University, Maslak 34469, Istanbul, Turkey \\ ${ }^{3}$ Physics Department and CICECO Materials Institute of Aveiro, University of Aveiro, 3810193 Aveiro, \\ Portugal
}

(Received 12 May 2016; accepted 25 August 2016; published online 4 October 2016)

\begin{abstract}
The natural ability of peptides and proteins to self-assemble into elongated fibrils is associated with several neurogenerative diseases. Diphenylalanine (FF) tubular structures that have the same structural motif as in A $\beta$-amyloid peptide (involved in Alzheimer's disease) are shown to possess remarkable physical properties ranging from piezoelectricity to electrochemical activities. In this work, we also discover a significant pyroelectric activity and measure the temperature dependence of the pyroelectric coefficient in the temperature range of $20100{ }^{\circ} \mathrm{C}$. Pyroelectric activity decreases with temperature contrary to most ferroelectric materials and significant relaxation of pyrocurrent is observed on cooling after heating above $50{ }^{\circ} \mathrm{C}$. This unusual behavior is assigned to the temperature-induced disorder of water molecules inside the nanochannels. Pyroelectric coefficient and current and voltage figures of merit are estimated and future applications of pyroelectric peptide nanostructures in biomedical applications are outlined. Published by AIP Publishing.
\end{abstract}

[http://dx.doi.org/10.1063/1.4962652]

Self-assembled bioorganic nanomaterials have attracted significant interest due to their emergent multifunctional properties derived from the unique structure and design variability offered by non-covalent interactions. ${ }^{1-3}$ Peptidebased nanostructures are of special importance, because they can be used in various applications ranging from biological scaffolds ${ }^{4}$ and templates for nanowire fabrications ${ }^{5}$ to lightemitting diodes ${ }^{6}$ and gates for field-effect transistors, ${ }^{7}$ and many more. The most studied self-organized peptide is diphenylalanine (FF) that combines useful physical properties with the ability of binding with several metals and other functional groups. It is derived from the core recognition motif of the Alzheimer's disease's A $\beta$-amyloid polypeptide thus being interesting from the biomedical point of view. It has been shown that FF-based materials are prone to selfassembly into tubular, ${ }^{8}$ spherical, ${ }^{9}$ rod-like, ${ }^{10}$ and fibrous structures ${ }^{11}$ depending on the deposition method and solvent chemistry. Excellent mechanical, ${ }^{12}$ electric, ${ }^{13}$ piezoelectric, ${ }^{14}$ optical, ${ }^{6,15}$ and electrochemical ${ }^{16}$ properties have demonstrated great potential of using FF-structures in nanoand micro-devices. The existence of temperature-dependent spontaneous polarization naturally suggests the possibility of pyroelectric response $^{17}$ that is a common feature of many natural biological materials. ${ }^{18}$ Recent interest to nanoscale pyroelectric materials is fueled by the expectations of the significant increase of pyroelectric coefficients upon miniaturization $^{19}$ and various possibilities for thermal energy harvesting. ${ }^{20}$ Recent studies by the group of Wang have opened new perspectives for $\mathrm{ZnO}$-based nanogenerators with high conversion efficiency of about $0.05 \mathrm{Vm}^{2} / \mathrm{W} .^{21}$ Peptides with their intrinsic biocompatibility and low thermal mass could

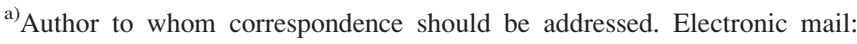
kholkin@ua.pt
}

be a viable alternative of these in biomedical applications. In this work, we explore these possibilities.

The lyophilized form of FF peptide (H-D-Phe-DPhe-OH, FF) was purchased from Bachem (Bubendorf, Switzerland). The solvent 1,1,1,3,3,3-hexafluoro-2-propanol (HFP) was used to prepare the solution (Sigma-Aldrich, Germany). Fresh peptide stock solution was prepared by dissolving the peptide powder in HFP at a concentration of $100 \mathrm{mg} / \mathrm{ml}$. To avoid any pre-aggregation, stock solutions were prepared before each experiment. The FF peptide stock solution was then diluted with deionized water $(\mathrm{pH} 6.67)$ to a final concentration of $2 \mathrm{mg} / \mathrm{ml}$.

In this work, we used the following method of sample preparation: ${ }^{22}$ first, $2 \mu \mathrm{l}$ of FF stock solution were applied to the substrate and then $98 \mu \mathrm{l}$ of water were added. The resulting drops were dried at room temperature for one day. Sufficiently big FF peptide microtubes grown from the same spot on the microdrop boundary were collected from the $\mathrm{Pt}$ substrate and used for experiments. ${ }^{21}$ The diameters of the individual microtubes were in the range of $13 \mu \mathrm{m}$ and lengths up to $1 \mathrm{~mm}$ (supplementary material). In order to increase the current, many microtubes were assembled as bundles and manually placed in a special holder, where the bundles were suspended between the two needle electrodes and fixed with a silver paste to ensure good electrical and mechanical contacts [Fig. 1(a)]. The electrodes were clamped between the two metal frames with Teflon spacers. Such a structure was used to suppress the background current down to a fA level.

Figure 1(b) shows a schematic of the measurement system (modified Chynoweth setup ${ }^{23}$ ). Microtubes were periodically heated by the pulses of defocused irradiation from a $\mathrm{CW} 10.6 \mu \mathrm{m} \mathrm{CO} 2$ laser with a maximum power of $40 \mathrm{~W}$ (VL300, VersaLaser). Laser intensity was modulated by the 

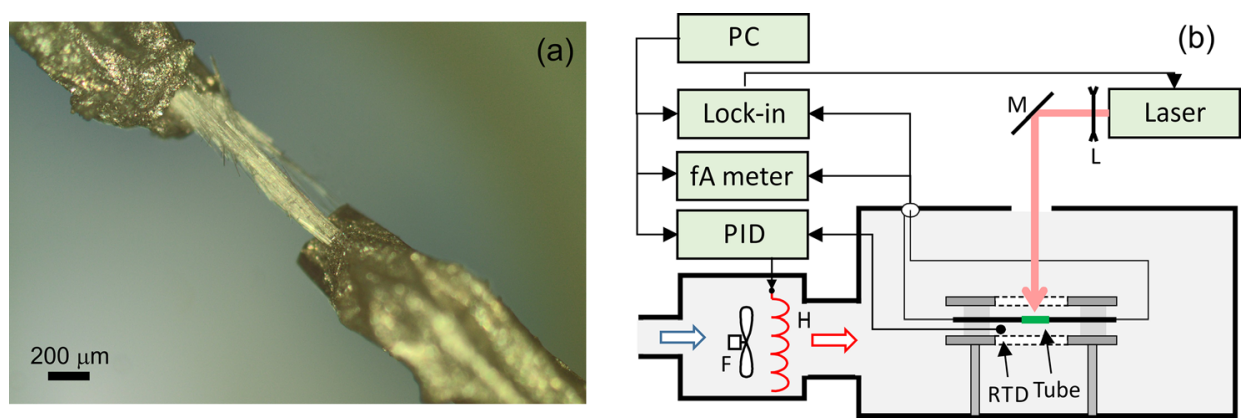

FIG. 1. (a) Optical image of the FF microtube bundle fixed between two electrodes by silver paste and (b) sche matic of the measurement setup. $\mathrm{F}$ is the fan, $\mathrm{H}$ is the heater, and PID is the tem perature controller. Fan vibrations did not influence the pyroelectric current measurements because of the high selec tivity of lock in amplifier.

square waveform either with a variable frequency directly from the lock-in internal reference TTL output (with 50\% duty-cycle) or with a variable duty-cycle by multifunctional data acquisition board (PCI 6251, National Instruments) synchronously triggered by the lock-in internal reference. The rise- and fall-times of the laser output power were $120 \pm 40 \mu$ s. Pyroelectric current was registered either by the lock-in amplifier (SR-830A, Stanford Research Systems) using its current input or by the sub-fA meter (6430, Keithley) with remote current preamplifier. The frequency dependence of pyroelectric current was studied using a 50\% duty cycle to maintain the same average power intensity on the sample.

Figure 2 shows a representative current waveform obtained under irradiation with a sufficiently long laser pulse $(\approx 1 \mathrm{~s})$. A typical pyroelectric-like response was observed: namely, switching the laser on (heating) was accompanied by the positive current spike, while switching the laser off (cooling) resulted in the negative peak. It should be noted that the input frequency bandwidth of the fA meter and sampling rate $(\sim 240$ points/s) were rather limited as compared to the fast rise-time and fall-time of the laser source. Moreover, input bandwidth was shown to vary significantly with the selected current limit and filtering parameters. Thus, such measurements did not allow obtaining quantitative data on the amplitude and shape of the current pulse as well as verifying that the pyroelectric current $I$ is indeed proportional to the temperature time derivative, i.e., $I \sim \partial T / \partial t$.

In order to verify the nature of the response and rule out possible parasitic effects (e.g., thermoelectric contribution or electro-magnetic interference), we measured the current

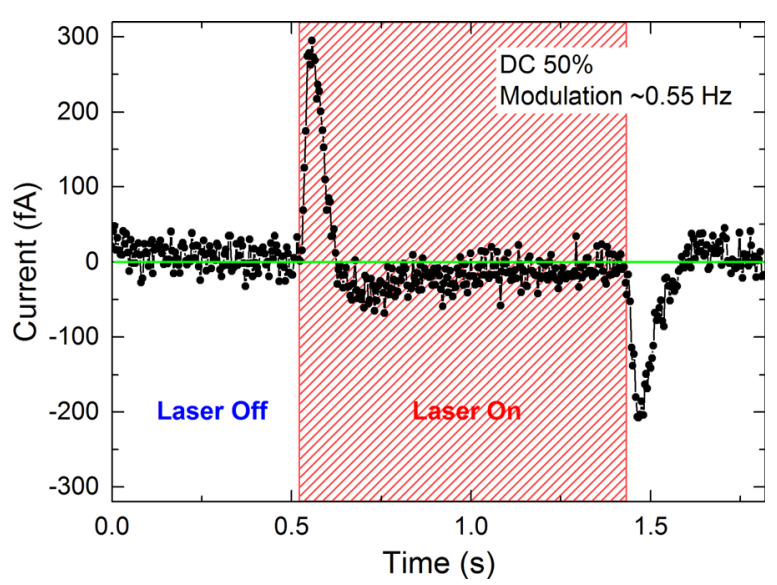

FIG. 2. Representative waveform of the pyroelectric current of the FF microtubes bundle under irradiation with a $\mathrm{CO}_{2}$ laser with the duty cycle of $50 \%$ measured by fA meter at room temperature. from a dummy sample (no microtube between the electrodes) and frequency dependence of the response. No measurable current was detected for the dummy sample. Figure 3 shows the frequency dependence of the pyroelectric current measured by the lock-in amplifier having a frequency bandwidth of $70 \mathrm{kHz}$ for the used current input.

As can be seen from Fig. 3, pyroelectric current significantly increases with increasing frequency following the expected dependence, when the temperature modulation frequency is much smaller than the thermal relaxation time $\tau=C / G$ (where $C$ is the heat capacitance and $G$ is the thermal conductivity of a microtube bundle). We can estimate $\tau=2 \pi / f \approx 30 \mathrm{~ms}$ based on the frequency $f \approx 200 \mathrm{~Hz}$, at which the current is almost saturated (Fig. 3). In order to estimate the pyroelectric coefficient at room temperature, we used the well-known equation (valid for $f \geq 2 \pi / \tau$ ),

$$
I=\frac{A \gamma p W_{o}}{C},
$$

where $A$ is the electrode area, $\gamma$ is the ratio between the absorbed and incident power, $p$ is the pyroelectric coefficient, and $W_{o}$ is the incoming power from the laser.

Assuming that the incident power with the estimated density of about $20 \mathrm{~kW} / \mathrm{m}^{2}$ is fully absorbed by the microtube bundle (i.e., $\gamma=1$ ) and taking the value of specific heat for the microtubes measured by differential scanning calorimetry $\left(\mathrm{C}=1871 \mathrm{~J} /(\mathrm{kg} \mathrm{K})^{24}\right)$, we obtain the pyroelectric coefficient $p \geq 2 \mu \mathrm{C} /\left(\mathrm{m}^{2} \mathrm{~K}\right)$. We should stress that this is a lower limit of the calculated pyroelectric coefficient because

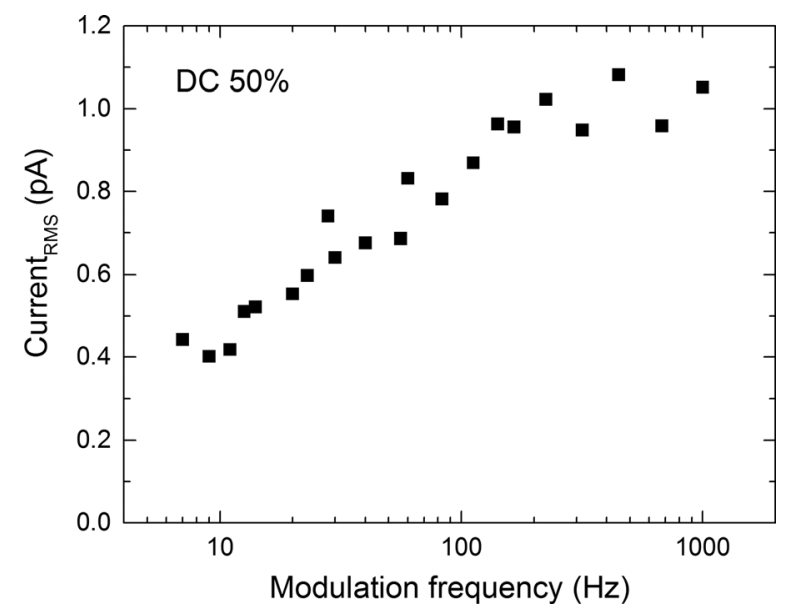

FIG. 3. Frequency dependence of the RMS amplitude of pyroelectric current under illumination with $\mathrm{CO}_{2}$ laser with the duty cycle (DC) of $50 \%$ mea sured by the lock in amplifier at room temperature. 
of the uncertainties in the evaluation of reflection coefficient. This value is about one order of magnitude smaller than that of the well-known polymer pyroelectric poly(vinylidene fluoride-trifluoroethylene) [P(VDF-TrFE)], but it is comparable with those of pyroelectric wide-band semiconductors, such as AlN, CdS, and GaN. ${ }^{25}$ We estimated the lower limits of current and voltage figures of merit for pyroelectric effect as $F_{i}>0.91 \times 10^{12} \mathrm{~m} / \mathrm{V}$ and $F_{v}>0.026 \mathrm{~m}^{2} / \mathrm{C}$ thus confirming their usefulness for pyroelectric energy harvesting. ${ }^{35}$ Voltage figure of merit is close to that of AlN or $\mathrm{ZnO}$ (supplementary material). We also expect that the pyroelectric response in nanotubes could be significantly increased as compared to that in microtube bundles due confinement effects ${ }^{18}$ and possibility of pyroelectric current compensation because polarization of individual microtubes could be oppositely oriented in the bundles. The advantage of pyroelectric self-assembled peptides as compared to inorganic nanomaterials such as $\mathrm{AlN}$ or $\mathrm{ZnO}$ is their easy functionalization and possibility to generate electric signals in biological environment at nanoscale dimensions.

One of the salient features of the ferroelectric pyroelectrics is their ability to increase the pyroelectric sensitivity while approaching the phase transition temperature between ferroelectric and paraelectric phases. We measured the temperature dependence of the pyroelectric current in FF microtubes to verify the possibility of approaching the ferroelectric phase transition ${ }^{26}$ at elevated temperatures by heating the ambient air in the enclosure. A typical result is shown in Fig. 4.

It can be seen that the pyroelectric current amplitude gradually decreases with temperature in contradiction with the earlier optical second harmonic and piezoelectric data showing a gradual decay of polarization with temperature. ${ }^{27,28}$ Heating to the temperature of about $4550^{\circ} \mathrm{C}$ and following cooling showed almost reversible behavior of the pyroelectric current. On the contrary, at higher maximum temperature achieved within the temperature cycle, the irreversible behavior of the pyroelectric current amplitude is noted. Moreover, the value of pyroelectric current on cooling

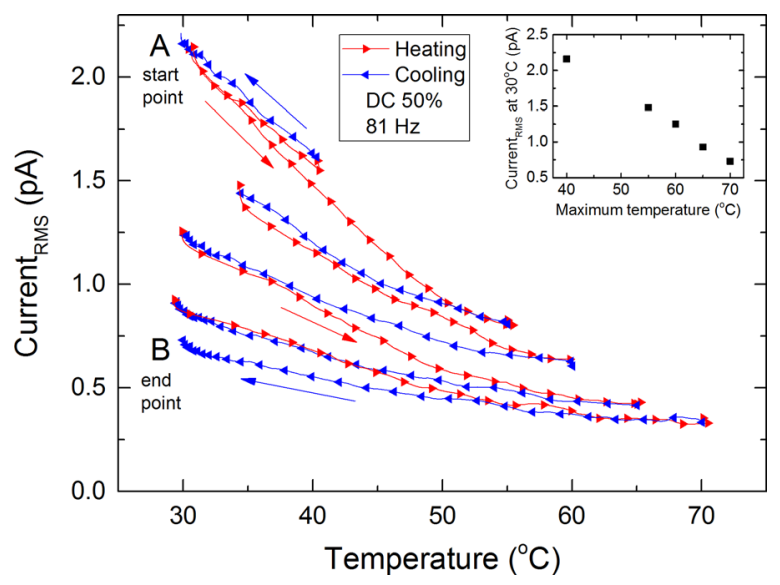

FIG. 4. Temperature dependence of the pyroelectric current (RMS value) in the FF microtubes measured by the lock in amplifier under consecutive heat ing/cooling cycles with the gradually increasing (from A to B) maximum temperature. Red curves are related to heating, while the blue ones denote heating after cooling. Inset displays pyroelectric current at $30^{\circ} \mathrm{C}$ as a func tion of maximum heating temperature. is always smaller than on heating within the same cycle. The behavior of the current as a function of maximum temperature is shown in the inset to Fig. 4. It is believed that such a decrease in the pyroelectric current may originate from the combined heating action of the laser itself and the external heater. The estimated increase in the temperature of the microtubes due to laser heating could be greater than $5^{\circ} \mathrm{C}$. Such temperature increase could influence the degree of ordering of water molecules existing in the nanochannels of individual nanotubes composing a microtube. ${ }^{29}$ The hydrophilic interactions of water with $\mathrm{COO}$ tails are temperaturedependent and the disruption of dipole ordering occurs mainly in the inner core of water structure. ${ }^{30}$ Therefore, the dipolar order of water molecules located near carboxyl groups in the nanocavities cannot recover at these temperatures due to their high mobility resulting in a gradual decrease of pyroelectric current (Fig. 4). The fact that the pyroelectric current on cooling is always smaller than on heating indicates a lack of order recovery which is a salient feature of 1D water in the FF nanochannel. However, once stabilized by heating to high temperature, pyroelectric properties of the microtubes are stable and can be used at lower temperatures.

The device application of the discovered pyroelectric properties of FF nano- and microtubes can be two-fold. First, the generated current proportional to temperature variation can be used as a nanoscale temperature probe (thermometer) compatible with the biological environment. Such probes based on luminescence ${ }^{31}$ and thermoelectric ${ }^{32}$ properties of nanostructures have been already reported. The advantage of using pyroelectric thermometers can be their sensitivity to the temperature variation rather than to temperature itself. It helps, for example, to monitor fast thermal properties in the cells. ${ }^{33}$ Another envisaged application is in the area of thermal energy harvesting, which may compete with the piezoelectric harvesting when the sudden variations of temperature are found, e.g., in the human/animal body. These variations may scavenge the energy in the microwatt range, which might be enough due to a precipitous decline in the power consumption of microelectronics. ${ }^{34}$ In this context, peptide nano- and microtubes provide various possibilities due to their enormous flexibility in the design: from the "nanoforest" obtained by physical vapor deposition ${ }^{35}$ to epitaxial films. ${ }^{36}$ Pyroelectric nanogenerators can be easily prepared using the same design as for the $\mathrm{ZnO}$-based harvesters. ${ }^{21} \mathrm{We}$ argue that somewhat lower pyroelectric coefficients in FF peptides can be compensated by their high biological functionality and possibilities offered by bionanotechology. As such, they can be used as pyroelectric energy harvesters or temperature sensors similar to the nanostructured $\mathrm{ZnO}^{21}$ Once long-term implantable electricity harvesters are available, they could enable tiny distributed devices to stimulate nerves and alleviate chronic pain. ${ }^{37}$ Thus, the observed pyroelectric effect in the FF microtubes could find a multitude of applications in biomedical devices.

To summarize, we discovered a notable pyroelectric effect in the FF microtubes grown from the solution. The pyroelectric coefficient is in excess of $2 \mu \mathrm{C} /\left(\mathrm{m}^{2} \mathrm{~K}\right)$ and is comparable to that of common semiconductor pyroelectrics, such as AlN or $\mathrm{ZnO}$. The pyroelectric current amplitude 
decreases with increasing temperature and increases with frequency. Pyroelectric voltage voltage responsivity is sufficiently high rendering this material for a multitude of biomedical applications.

See supplementary material for the representative SEM image of the individual FF microtube and comparison of pyroelectric figures of merit for different classes of materials including FF.

F.S. and A.K. thank the Turkish-Portuguese Project No. TUBITAK/0006/2014 for the financial support. A.K. acknowledges CICECO-Aveiro Institute of Materials (Ref. FCT UID/CTM/50011/2013) financed by national funds through the FCT/MEC and, when applicable, co-financed by FEDER under the PT2020 Partnership Agreement. S.V. was supported by the President of Russian Federation grant for young scientists (Contract No. 14.Y30.15.6554-MK). The equipment of the Ural Center for Shared Use "Modern Nanotechnology" of Ural Federal University was used.

${ }^{1}$ K. Ariga, T. Mori, and J. P. Hill, Adv. Mater. 24, 158 (2012).

${ }^{2}$ C. Valery, F. Artzner, and M. Paternostre, Soft Matter 7, 9583 (2011).

${ }^{3}$ L. Adler Abramovich and E. Gazit, Chem. Soc. Rev. 43, 6881 (2014).

${ }^{4}$ Y. Yanlian, K. Ulung, W. Xiume, A. Horii, H. Yokoi, and Z. Shuguang, Nano Today 4, 193 (2009).

${ }^{5}$ M. Yemini, M. Reches, J. Rishpon, and E. Gazit, Nano Lett. 5, 183 (2005).

${ }^{6}$ J. Ryu, S. Y. Lim, and C. B. Park, Adv. Mater. 21, 1577 (2009).

${ }^{7}$ T. Cipriano, G. Knotts, A. Laudari, R. C. Bianchi, W. A. Alves, and S. Guha, ACS Appl. Mater. Interfaces 6, 21408 (2014).

${ }^{8} \mathrm{M}$. Reches and E. Gazit, Science 300, 625 (2003).

${ }^{9}$ M. Reches and E. Gazit, Nano Lett. 4, 581 (2004).

${ }^{10}$ Q. Li, Y. Jia, L. Dai, Y. Yang, and J. Li, ACS Nano 9, 2689 (2015).

${ }^{11}$ J. Kim, T. H. Han, Y. L. Kim, S. Park, J. Choi, D. G. Churchill, S. O. Kim, and $\mathrm{H}$. Ihee, Adv. Mater. 22, 583 (2010).

${ }^{12}$ L. Adler Abramovich, N. Kol, I. Yanai, D. Barlam, R. Z. Shneck, E. Gazit, and I. Rousso, Angew. Chem. Int. Ed. 49, 9939 (2010).

${ }^{13}$ M. Wang, L. Du, X. Wu, S. Xiong, and P. K. Chu, ACS Nano 5, 4448 (2011).
${ }^{14}$ A. L. Kholkin, N. Amdursky, I. Bdikin, G. Rosenman, and E. Gazit, ACS Nano 4, 610 (2010).

${ }^{15}$ T. Nikitin, S. Kopyl, V. Ya. Shur, Y. V. Kopelevich, and A. L. Kholkin, Phys. Lett. A 380, 1658 (2016).

${ }^{16}$ R. De La Rica and H. Matsui, Chem. Soc. Rev. 39, 3499 (2010).

${ }^{17}$ R. Whatmore, Rep. Prog. Phys. 49, 1335 (1986).

${ }^{18}$ S. B. Lang, Nature 212, 704 (1966).

${ }^{19}$ A. N. Morozovska, E. A. Eliseev, G. S. Svechnikov, and S. V. Kalinin, J. Appl. Phys. 108, 042009 (2010).

${ }^{20}$ C. R. Bowen, J. Taylor, E. LeBoulbar, D. Zabek, A. Chauhan, and R. Vaish, Energy Environ. Sci. 7, 3836 (2014).

${ }^{21}$ Y. Yang, W. Guo, K. C. Pradel, G. Zhu, Y. Zhou, Y. Hu, L. Lin, and Z. L. Wang, Nano Lett. 12, 2833 (2012).

${ }^{22}$ A. Nuraeva, S. Vasilev, D. Vasileva, P. Zelenovskiy, D. Chezganov, A. Esin, S. Kopyl, K. Romanyuk, V. Ya. Shur, and A. L. Kholkin, Cryst. Growth Des. 16, 1472 (2016).

${ }^{23}$ A. G. Chynoweth, J. Appl. Phys. 27, 78 (1956).

${ }^{24}$ S. Kopyl, private communication (2016).

${ }^{25}$ C. R. Bowen, H. A. Kim, P. M. Weaver, and S. Dunn, Energy Environ. Sci. 7, 25 (2014).

${ }^{26}$ Z. Gan, X. Wu, X. Zhu, and J. Shen, Angew. Chem. Int. Ed. 52, 2055 (2013).

${ }^{27}$ A. Heredia, I. Bdikin, S. Kopyl, E. Mishina, S. Semin, A. Sigov, K. German, V. Bystrov, and A. L. Kholkin, J. Phys. D 43, 462001 (2010).

${ }^{28}$ S. Vasilev, P. Zelenovskiy, D. Vasileva, A. Nuraeva, V. Y. Shur, and A. L. Kholkin, J. Phys. Chem. Sol. 93, 68 (2016).

${ }^{29}$ P. M. G. L. Ferreira, M. S. Ishikawa, S. Kogikoski, Jr., W. A. Alves, and H. Martinho, Phys. Chem. Chem. Phys. 17, 32126 (2015).

${ }^{30}$ Z. Luo, B. Akerman, S. Zhang, and B. Norden, Soft Matter 6, 2260 (2010).

${ }^{31}$ C. D. S. Brites, P. P. Lima, N. J. O. Silva, A. Millan, V. S. Amaral, F. Palacio, and L. D. Carlos, Nanoscale 4, 4799 (2012).

${ }^{32}$ P. C. Fletcher, B. Lee, and W. P. King, Nanotechnology 23, 035401 (2012).

${ }^{33}$ S. Arai, S. C. Lee, D. Zhai, M. Suzuki, and Y. T. Chang, Sci. Rep. 4, 6701 (2014).

${ }^{34}$ R. P. Lopes and A. L. Kholkin, in Energy Harvesting with Piezoelectric and Pyroelectric Materials, edited by N. Muensit (Trans Tech Publications, Switzerland, 2011), pp. 122140.

${ }^{35}$ L. Adler Abramovich, D. Aronov, P. Beker, M. Yevnin, S. Stempler, G. Rosenman, and E. Gazit, Nat. Nanotechnol. 4, 849 (2009).

${ }^{36}$ V. Nguyen, K. Jenkins, and R. Yang, Nano Energy 17, 323 (2015).

${ }^{37}$ S. E. Moulton, M. J. Higgins, R. M. I. Kapsa, and G. G. Wallace, Adv. Funct. Mater. 22, 2003 (2012). 